\title{
Assessment of the Efficacy of Rifaximin in the Management of Irritable Bowel Syndrome (IBS)
}

\author{
Stanislas A. Doffou', Kissi Henriette Anzouan-Kacou', Eugene K. Messou' ${ }^{2}$, Demba A. Bangoura ${ }^{1}$, \\ Dimitri H. Kouamé1, Kouame M. Alassan'1, Constant Assi ${ }^{3}$, Fulgence Mamert Y. Bathaix ${ }^{1}$, \\ Alain K. Attia1, Marie-Jeanne K. Lohoues ${ }^{3}$
}

\footnotetext{
${ }^{1}$ Yopougon University Hospital, Abidjan, Côte d'Ivoire

${ }^{2}$ Treichville University Hospital, Abidjan, Côte d'Ivoire

${ }^{3}$ Cocody University Hospital, Abidjan, Côte d'Ivoire

Email: das_stan@yahoo.ca
}

How to cite this paper: Doffou, S.A., Anzouan-Kacou, K.H., Messou, E.K., Bangoura, D.A., Kouamé, D.H., Alassan, K.M., Assi, C., Bathaix, F.M.Y., Attia, A.K. and Lohoues, M.-J.K. (2017) Assessment of the Efficacy of Rifaximin in the Management of Irritable Bowel Syndrome (IBS). Open Journal of Gastroenterology, 7, 261-270. https://doi.org/10.4236/ojgas.2017.710027

Received: September 18, 2017

Accepted: October 28, 2017

Published: October 31, 2017

Copyright $\odot 2017$ by authors and Scientific Research Publishing Inc. This work is licensed under the Creative Commons Attribution International License (CC BY 4.0).

http://creativecommons.org/licenses/by/4.0/

\begin{abstract}
The aim of our study was to assess in our context, the efficacy of Rifaxim in improving the symptoms of irritable bowel syndrome particularly in its diarrheal (IBS-D) or mixed (IBS-DC) component and therefore assess its impact on patients' quality of life. Patients and methods: This was an uncontrolled, non-comparative prospective cohort study of a single group of patients. $\mathrm{Pa}$ tients recruitment was done in two University Hospitals for 6 months (September 2015-February 2016). Were included ambulatory patients, male or female, aged 18 - 75 years, with diarrheic IBS (IBS-D) or mixed IBS (IBS-DC) diagnosed according to Rome III criteria and who agreed to participate in the study. Each patient received 400mg Rifaximin $\times 2 / \mathrm{d}$ for 15 days. The overall assessment of the efficacy of treatment at D15 (end of treatment) and D30 (2 weeks post-treatment) was the primary criterion of judgment. The statistical tests used were the Chi-square test and Fisher's exact test for the qualitative variables and Student's test for the quantitative variables. Results: A total number of 30 patients ( 16 women) with an average age of $44.5 \pm 13.9$ years were included. The overall assessment of symptoms by the patient with the Likert scale found $28(93.3 \%)$ patients, $12(40 \%)$ patients and 10 patients (33.3\%) for a scale $\geq 2$ at D0, D15 and D30 respectively. The assessment of the intensity of pain or abdominal discomfort found at D0, D15 and D30 respectively: 27(90\%), 13(43.4\%) and 6(20.1\%) patients who had an EVA score $>2$. The mean score for Francis to assess the improved quality of life was $247.1 \pm 97.4$ at D0, $99.8 \pm 63.1$ at D15 and $128.8 \pm 70.6$ at D30. Conclusion: There is a good overall improvement of symptoms in our patients suffering from IBS-D or mixed (IBS-DC) on Rifaximin with improvement of the quality of life.
\end{abstract}




\section{Keywords}

Rifaximin, Irritable Bowel, Diarrhea, Quality of Life

\section{Introduction}

Irritable Bowel Syndrome is a common condition. Although benign, the chronic and recurring nature of symptoms has a strong impact on patients' quality of life. This impairment of quality of life is as important as that noted in insulin-dependent diabetes or chronic renal failure [1]. Intestinal functional disorders remain a public health problem. Irritable Bowel Syndrome (IBS) is a multifactorial disease, and better understanding of the pathophysiological mechanisms of IBS has led to a progressive diversification of management options. Among the possible pathophysiological approaches, more and more data highlight the role played by abnormalities of the intestinal microbiota in the onset of symptoms [2]. This evolution in the pathophysiological design of the IBS opens new therapeutic perspectives with the possible use of probiotics, prebiotics and antibiotics such as Rifaximin to improve the digestive comfort and quality of life of persons suffering from IBS [3].

During irritable bowel syndrome (IBS), the primary objective of treatment is to improve the abdominal pain or chronic discomfort that bowel movements usually temporally relieve. This is the main complaint that leads the patient to consult. The improvement in the quality of life of the patients also involves an improvement in the transit disorders constipation (IBS-C), diarrhea (IBS-D) or alternation of both (IBS-A) that are constant and more marked during painful surges [1]

The aim of this study was to assess the efficacy of Rifaximin in improving the symptoms of irritable bowel syndrome, particularly in its diarrheic (IBS-D) or mixed (IBS-DC) component and therefore assess its impact on the quality of life of patients.

\section{Patients and Methods}

This was an uncontrolled, non-comparative prospective cohort study of a single group of patients. It was carried out from July 2016 to October 2016 in the Hepato-Gastro-Enterology departments of Yopougon and Cocody University Hospitals.

Were included in this study all ambulatory patients, both male and female (non-pregnant and non-nursing), aged 18 - 75 years, who had irritable bowel syndrome of (diarrhea-type IBS-D) or of mixed type (IBS-DC) defined according to Rome III criteria with pain or abdominal discomfort. Were not included patients who had an IBS of type constipation only (according to Rome III criteria), who presented warning signs (anemia, rectal bleeding, unexplained weight loss, general condition impairment), history of Gastrointestinal cancer or ga- 
strointestinal surgery (other than cholecystectomy or appendectomy) or systemic pathology (collagenosis, diabetes mellitus), history of inflammatory bowel disease, unstable thyroid disease, HIV and renal or hepatic impairment. Patients were also not included if they took anti-psychotic, anti-spasmodic, anti-diarrheal, probiotic or narcotic medications, or had taken antibiotics in the previous 14 days or Rifaximin within 30 days prior to inclusion. An informed and signed consent was obtained from each participant prior to their entering the study. Each patient received $800 \mathrm{mg}$ of Rifaximin per day $(400 \mathrm{mg} \times 2 / \mathrm{d})$ for 14 days. The assessment of the efficacy of treatment on symptoms was assessed by the patient himself at the end of the 14-day treatment (D15) and 2 weeks after the end of treatment (D30) in comparison with the symptomatology before inclusion (D0). The main criterion is the overall assessment of treatment with the 5-point Likert scale. Treatment is judged to be effective if the Likert corresponds to 0 or 1 . Secondary criteria are the improvement of the quality of life through a standardized questionnaire of quality of life (Francis score), the evolution of the intensity of pain or abdominal discomfort by a VAS score (visual analog scale), diarrhea assessment by the number of stools produced per week, and assessment of stool consistency according to the Bristol scale. The quality of life is considered better if Francis score is less than 176 and the abdominal pain or discomfort is considered acceptable if VAS less than 2. Only data from patients with good compliance were taken into account. This was obtained by an oral interview of each patient.

All these data were collected on individual survey forms, entered and processed by the Excel software. The statistical analysis of the factors studied was performed by a Chi square test and the Fisher Exact test of Pearson to compare qualitative variables with a $p<0.05$ coefficient of significance. The comparison of quantitative variables (mean and median) was performed by an analysis of variance (ANOVA).

\section{Results}

Thirty-three patients were included in the 3 months of our study. For the analysis, only data from 30 patients were used, 3 did not comply with the treatment correctly.

The average age of our patients was 44.5 years $( \pm 13.9)$. There was a slight female predominance $(53.3 \%)$. Only $2(6.7 \%)$ of the patients were smokers and 3 (10\%) had NSAIDs prior to inclusion. At baseline, 28 patients (93.3\%) experienced moderate to severe symptoms (Likert $\geq 2$ ), 27 (90\%) complained of abdominal pain or discomfort at least moderately (VAS > 2). Almost all patients (96.7\%) had an abnormal stool appearance on the Bristol scale with an average stool per week at $9.1 \pm 4.6$. Almost $2 / 3$ of the patients had a quality of life impaired by symptoms (Francis score $\geq 176$ ) with an average Francis score of 247.1 \pm 97.4 at D0. The general features of patients at baseline are summarized in $\mathrm{Ta}$ ble 1 . 
Table 1. General features of patients at baseline (D0).

\begin{tabular}{|c|c|c|}
\hline Diarrhea-abdominal pain & 7 & $(23.3 \%)$ \\
\hline Female gender & 16 & $(53.3 \%)$ \\
\hline Average age in years (ET) & 44.5 & $(13.9)$ \\
\hline Median age (IBC) & 47 & $(31-50)$ \\
\hline Married & 13 & $(43.3 \%)$ \\
\hline Alcohol comsomption & 15 & $(50 \%)$ \\
\hline Tobacco use & 2 & $(6.7 \%)$ \\
\hline NSAIDs* taking & 3 & $(10 \%)$ \\
\hline Anti-depressant intake & 1 & $(3.3 \%)$ \\
\hline Anti-spasmodic intake & 9 & $(30 \%)$ \\
\hline Anti-diarrheal intake & 9 & $(30 \%)$ \\
\hline Antibiotic intake & 9 & $(30 \%)$ \\
\hline Presence of stress & 13 & $(43.3 \%)$ \\
\hline Likert: $\geq 2(\%)$ & 28 & $(93.3 \%)$ \\
\hline $\mathrm{VAS}^{* *}>2(\%)$ & 27 & $(90 \%)$ \\
\hline Diarrhea-constipation & 14 & $(46.7 \%)$ \\
\hline Abnormal stool appearance according to Bristol (\%) & 29 & $(96.7 \%)$ \\
\hline Worsening of signs by stress (\%) & 14 & $(46.7 \%)$ \\
\hline Francis score $\geq 176(\%)$ & $20 / 29$ & $(69 \%)$ \\
\hline Average number of stools/week at D0 (ET) & 9.1 & $(4.6)$ \\
\hline Average $\mathrm{VAS}^{* *}$ at $\mathrm{D} 0(\mathrm{ET})$ & 5.6 & $(2.3)$ \\
\hline Average Francis score of at D0 (ET) & 247.1 & $(97.4)$ \\
\hline
\end{tabular}

${ }^{*}$ Non Steroid Anti-Inflammatory Drugs; ${ }^{* *}$ Visual Analog Scale.

An overall symptom improvement was observed with a Likert scale $\geq 2$ at D15 and 30 respectively in $40 \%$ and $33.3 \%$ of patients (Figure 1). Abdominal pain or discomfort had regressed with an average EVA score of $2.5 \pm 1.9$ at D15 and $2 \pm$ 2 at D30 instead of $5.6 \pm 2.3$ at D0. The stool appearance was not normal only in 10 patients (33.3\%) at D15 and 12 patients (40\%) at D30 with respective averages/weeks of $6.8 \pm 2$ and $7.2 \pm 2$. 6. Quality of life improved in most patients with Francis mean scores of $99.8 \pm 63.1$ at D15 and $128 \pm 70.6$ at D30 (Figure 2). The comparative assessment of the variables studied at D0, D15 and D30 is summarized in Table 2.

\section{Discussion}

Irritable Bowel Syndrome (IBS) is a gastrointestinal syndrome characterized by chronic abdominal pain and altered intestinal habits without underlying organic pathology, diagnosed according to the revised ROME criteria [4]. Treatment of IBS is important because symptoms cause significant impairment of health-related 


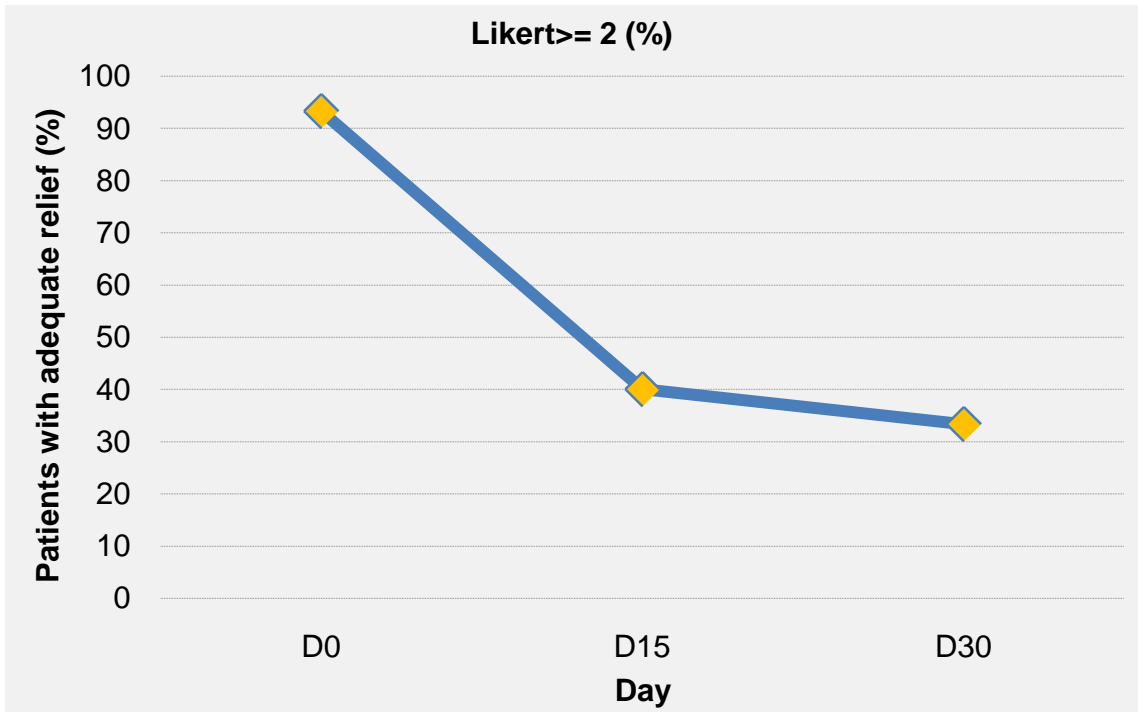

Figure 1. Evolution of global symptoms.

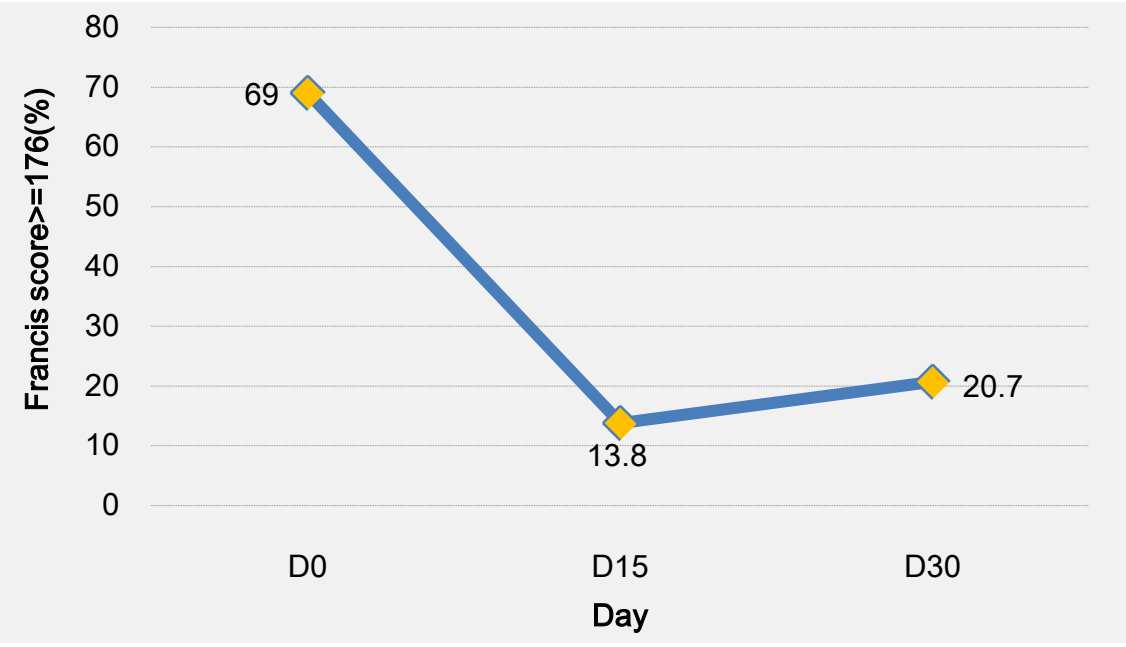

Figure 2. Quality of life evolution.

Table 2. Assessment of the variables at D0, D15 and D30.

\begin{tabular}{lccc}
\hline \multicolumn{1}{c}{ Variables } & D0 & D15 & D30 \\
\hline Likert $\geq 2$ (\%) & $28(93.3)$ & $12(40)$ & $10(33.3)$ \\
VAS $>$ 2 (\%) & $27(90)$ & $13(43.4)$ & $6(20.1)$ \\
Diarrhea-constipation (\%) & $16(30)$ & $4(13.3)$ & $5(16.7)$ \\
Diarrhea (\%) & $26(86.7)$ & $10(33.3)$ & $6(20)$ \\
Abnormal appearance of stools & $29(96.7)$ & $10(33.3)$ & $12(40)$ \\
according to Bristol (\%) & $14(46.7)$ & $10(33.3)$ & $5(16.7)$ \\
Worsening of signs/stress (\%) & $20 / 29(69)$ & $4 / 29(13.8)$ & $6 / 29(20.7)$ \\
Francis score $\geq 176$ (\%) & $9.1(4.6)$ & $6.8(2)$ & $7.2(2.6)$ \\
Average number of stools/week (ET) & $5.6(2.3)$ & $2.5(1.9)$ & $2(2)$ \\
Average VAS (ET) & $247.1(97.4)$ & $99.8(63.1)$ & $128.8(70.6)$ \\
Average francis score (ET) & & & \\
\hline
\end{tabular}


quality of life, resulting in increased use of health resources and reduced labor productivity [5] [6] [7] [8]. The intestinal microbiota has been explored as a treatment target in IBS, given its importance in the development of symptoms in some patients. Approaches include the use of antibiotics and probiotics. The best-studied antibiotic in IBS is Rifaximin [9]. It is a non-systemic antibiotic that appears to have anti-inflammatory activities, and modulation of the intestinal microbiota [10]-[22]. Rifaximin received regulatory approval for the treatment of IBS-D in May 2015 [23]. Several studies have revealed a favorable safety and efficacy profile for Rifaximin in IBS-D [24] [25] [26] [27].

Unlike most studies assessing Rifaximin, our work focused on a small sample (30 patients) without control group. The patient is himself his own judge at the end of the treatment of 14 days (D15) and 2 weeks after the end of treatment (D30). The primary assessment criterion was the proportion of patients who had adequate relief of IBS symptoms during treatment and 2 weeks after treatment. Our study showed an overall improvement in symptoms and quality of life in most of patients D15 and D30 compared to the pre-inclusion symptomatology with a dose of $800 \mathrm{mg} /$ day of Rifaximin. Patients did not feel or mildly felt symptoms in $60 \%$ and $67.67 \%$ respectively at D15 and D30 vs. $72 \%$ at D0. Pain or abdominal bloating was relieved in $87 \%$ of cases at D15 and in $79.9 \%$ of cases at $\mathrm{D} 30$ vs. $10 \%$ at $\mathrm{D} 0$. The results obtained in our work are in the same direction as several studies carried out elsewhere. Sharara et al. in a randomized double-blind, placebo-controlled trial showed superiority of Rifaximin in overall symptom relief ( $41.3 \%$ vs. $22.9 \%, p=0.03$ ) [28]. Another randomized, placebo-controlled study assessed the effect of Rifaximin $1200 \mathrm{mg} /$ day or placebo for 10 days in 87 patients with IBS. At the end of the 10-week follow-up period, patients receiving Rifaximin experienced a significantly higher overall improvement compared to the placebo group ( $p=0.02)$, with an average improvement of $36.4 \%$ in the Rifaximin group compared to $21.0 \%$ in the placebo group [25]. In addition, there was a significant improvement in bloating with Rifaximin, while abdominal pain, diarrhea and constipation did not change significantly in comparison with placebo.

The randomized, placebo-controlled TARGET 1 and 2 studies in phase III assessed identically studied the safety and efficacy of Rifaximin (550 mg 3 times daily) for 2 weeks in patients with IBS-D [26]. The primary efficacy criterion (the percentage of patients with adequate relief of the overall symptoms of IBS for $\geq 2$ of the first 4 weeks after the 2 -week treatment) was reached by $40.7 \%$ and $31.7 \%$ of patients receiving Rifaximin or placebo respectively $(p<0.001)$.

In addition, a significantly higher percentage of patients receiving Rifaximin had adequate relief from IBS-related bloating in the first 4 weeks after the 2-week treatment phase compared with placebo ( $40.2 \%$ versus $30.3 \%$, Respectively, $p<0.001)$. The results of these studies confirm that Rifaximin has a sustained effect in patients with IBS-D, with a 2 -week treatment cycle providing patients with a relief for at least 3 months [23]. In addition, three meta-analyzes of 
five randomized controlled trials of Rifaximin for IBS-D demonstrated a significant improvement in overall SBS symptoms with Rifaximin in comparison with placebo [29] [30] [31].

The antibiotic effect of Rifaximin is the presumed mechanism for its sustained beneficial effects in patients with IBS-D. A response to antibiotic therapy in patients with IBS has been shown to correlate with the standardization of lactulose hydrate allergy test results [28] [32]. However, there is a debate as to know which effect associated with the antibiotic is the most important. On the Basis of existing data, there are three reasonable explanations: Rifaximin affects intestinal bacteria and reduces bacterial products that negatively affect the host; the effect on the intestinal flora reduces the local engagement of the mucosa of bacteria such as the host's immune responses where the antibiotic modifies the bacteria and the response of the host. Regardless of the final approach, sustained effects suggest that Rifaximin affects an underlying cause of IBS that is related to an alteration of the intestinal microbiota [33] [34] [35] [36] Some patients in our study did not respond to treatment, which is consistent with the results of other placebo-controlled clinical trials involving patients with IBS [37] [38] [39] and which may reflect differences in the underlying cause of symptoms.

\section{Conclusion}

In our study, rifampicin at a dose of $800 \mathrm{mg} /$ day for 14 days improved the quality of life of patients suffering from IBS-D during treatment and at least 2 weeks later. But this assertion cannot be extended to all IBS-D patients given the small sample of patients included. It is therefore necessary to continue this work and extend it to a large number of patients.

\section{References}

[1] Coffin, B. (2013) Quel est l'impact du Syndrome de l'Intestin Irritable sur la qualité de vie des patients? [What Is the Impact of Irritable Bowel Syndrome on Patients' Quality of Life?] Hepato Gastro, 20, 11-15.

[2] Gourcerol, G., Melchior, C., Leroi, A.M. and Ducrotté, P. (2013) Traitement du syndrome de l'intestin irritable: où en est-on? [Management of Irritable Bowel Syndrome: Current Status and Future Perspective.] Hepato-Gastro, 20, 752-765.

[3] Ducroté, P. (2011) Microbiote et syndrome de l'intestin irritable. [Microbiota and Irritable Bowel Syndrome.] Lettre de l'hépato-gastroentérologue, XIV, 154-159.

[4] Longstreth, G.F., Thompson, W.G., Chey, W.D., Houghton, L.A., Mearin, F. and Spiller, R.C. (2006) Functional Bowel Disorders. Gastroenterology, 130, 1480-1491. https://doi.org/10.1053/j.gastro.2005.11.061

[5] Drossman, D.A., Li, Z., Andruzzi, E., et al. (1993) U.S. Householder Survey of Functional Gastrointestinal Disorders: Prevalence, Sociodemography, and Health Impact. Digestive Diseases and Sciences, 38, 1569-1580. https://doi.org/10.1007/BF01303162

[6] Camilleri, M. and, Andresen, V. (2009) Current and Novel Herapeutic Options for Irritable Bowel Syndrome Management. Digestive and Liver Disease, 41, 854-862. https://doi.org/10.1016/j.dld.2009.07.009 
[7] Hammerle, C.W. and Surawicz, C.M. (2008) Updates on Treatment of Irritable Bowel Syndrome. World Journal of Gastroenterology, 14, 2639-2649. https://doi.org/10.3748/wjg.14.2639

[8] El-Serag, H.B. (2003) Impact of Irritable Bowel Syndrome, Prevalence and Effect on Health Related Quality of Life. Reviews in Gastroenterological Disorders, 3, S3-S11.

[9] Lembo, A.J. (2015) New and Emerging Treatment Options for Irritable Bowel-Syndrome Current and Emerging Therapies for Irritable Bowel Syndrome. Gastroenterology \& Hepatology, 11, 9-15.

[10] Bajaj, J., Heuman, D., Sanyal, A., Hylemon, P., Sterling, R., Stravitz, R. et al. (2013) Modulation of the Metabiome by Rifaximin in Patients with Cirrhosis and Minimal Hepatic Encephalopathy. PLoS One, 8, e60042. https://doi.org/10.1371/journal.pone.0060042

[11] Brown, E., Xue, Q., Jiang, Z., Xu, Y. and DuPont, H. (2010) Pretreatment of Epithelial Cells with Rifaximin Alters Bacterial Attachment and Internalization Profiles. Antimicrob Agents Chemother, 54, 388-396.

https://doi.org/10.1128/AAC.00691-09

[12] Cheng, J., Shah, Y., Ma, X., Pang, X., Tanaka, T., Kodama, T., et al. (2010) Therapeutic Role of Rifaximin in Inflammatory Bowel Disease: Clinical Implication of Human Pregnane X Receptor Activation. Journal of Pharmacology and Experimental Therapeutics, 335, 32-41. https://doi.org/10.1124/jpet.110.170225

[13] Debbia, E., Maioli, E., Roveta, S. and Marchese, A. (2008) Effects of Rifaximin on Bacterial Virulence Mechanisms at Supra- and Sub-Inhibitory Concentrations. Journal of Chemotherapy, 20, 186-194. https://doi.org/10.1179/joc.2008.20.2.186

[14] Hopkins, K., Mushtaq, S., Richardson, J., Doumith,M., de Pinna, E., Cheasty, T., et al. (2014) In Vitro Activity of Rifaximin against Clinical Isolates of Escherichia coli and Other Enteropathogenic Bacteria Isolated from Travellers Returning to the UK. International Journal of Antimicrobial Agents, 45, 431-437.

[15] Jiang, Z., DuPont, H., La Rocco, M. and Garey, K. (2010) In Vitro Susceptibility of Clostridium difficile to Rifaximin and Rifampin in 359 Consecutive Isolates at a University Hospital in Houston, Texas. Journal of Clinical Pathology, 63, 355-358. https://doi.org/10.1136/jcp.2009.071688

[16] Jiang, Z., Ke, S. and DuPont, H. (2010) Rifaximininduced Alteration of Virulence of Diarrhoea-Producing Escherichia coli and Shigella sonnei. International Journal of Antimicrobial Agents, 35, 278-281.

[17] Maccaferri, S., Vitali, B., Klinder, A., Kolida, S., Ndagijimana, M., Laghi, L., et al. (2010) Rifaximin Modulates the Colonic Microbiota of Patients with Crohn's Disease: An in Vitro Approach using a Continuous Culture Colonic Model System. Journal of Antimicrobial Chemotherapy, 65, 2556-2565.

https://doi.org/10.1093/jac/dkq345

[18] Mencarelli, A., Migliorati, M., Barbanti, M., Cipriani,S., Palladino, G., Distrutti, E., et al. (2010) Pregnane-X-Receptor Mediates the Anti-Inflammatory Activities of Rifaximin on Detoxification Pathways in Intestinal Epithelial Cells. Biochemical Pharmacology, 80, 1700-1707.

[19] Mencarelli, A., Renga, B., Palladino, G., Claudio, D., Ricci, P., Distrutti, E., et al. (2011) Inhibition of NF-kB by a PXR-Dependent Pathway Mediates Counter-Regulatory Activities of Rifaximin on Innate Immunity in Intestinal Epithelial Cells. European Journal of Pharmacology, 668, 317-324.

[20] Schrodt, C., McHugh, E., Gawinowicz, M., DuPont, H. and Brown, E. (2013) Rifaximin-Mediated Changes to the Epithelial Cell Proteome: 2-D Gel Analysis. 
PLoS ONE, 8, e68550. https://doi.org/10.1371/journal.pone.0068550

[21] Terc, J., Hansen, A., Alston, L. and Hirota, S. (2014) Pregnane X Receptor Agonists Enhance Intestinal Epithelial Wound Healing and Repair of the Intestinal Barrier Following the Induction of Experimental Colitis. European Journal of Pharmaceutical Sciences, 55, 12-19.

[22] Xu, D., Gao, J., Gillilland, M., III, Wu, X., Song, I., Kao, J., et al. (2014) Rifaximin Alters Intestinal Bacteria and Prevents Stress-Induced Gut Inflammation and Visceral Hyperalgesia in Rats. Gastroenterology, 146, 484-496.

https://doi.org/10.1053/j.gastro.2013.10.026

[23] Foxx-Orenstein, A.E. (2016) New and Emerging Therapies for the Treatment of Irritable Bowel Syndrome: An Update for Gastroenterologists. Therapeutic Advances in Gastroenterology, 9, 354-375. https://doi.org/10.1177/1756283X16633050

[24] Di Stefano, M., Tana, P., Mengoli, C., Miceli, E., Pagani, E. and Corazza, G. (2011) Colonic Hypersensitivity Is a Major Determinant of the Efficacy of Bloating Treatment in Constipation-Predominant Irritable Bowel Syndrome. Internal and Emergency Medicine, 6, 403-411. https://doi.org/10.1007/s11739-010-0496-0

[25] Pimentel, M., Park, S., Mirocha, J., Kane, S.V. and Kong, Y. (2006) The Effect of a Nonabsorbed Oral Antibiotic (Rifaximin) on the Symptoms of the Irritable Bowel Syndrome: A Randomized Trial. Annals of Internal Medicine, 145, 557-563. https://doi.org/10.7326/0003-4819-145-8-200610170-00004

[26] Pimentel, M., Lembo, A., Chey, W., Zakko, S., Ringel, Y., Yu, J., et al. (2011) Target Study Group. Rifaximin Therapy for Patients with Irritable Bowel Syndrome without Constipation. The New England Journal of Medicine, 364, 22-32. https://doi.org/10.1056/NEJMoa1004409

[27] Pimentel, M., Chang, C., Chua, K., Mirocha, J., DiBaise, J., Rao, S., et al. (2014) Antibiotic Treatment of Constipation-Predominant Irritable Bowel Syndrome. Digestive Diseases and Sciences, 59, 1278-1285. https://doi.org/10.1007/s10620-014-3157-8

[28] Sharara, A.I., Aoun, E., Abdul-Baki, H., Mounzer, R., Sidani, S. and Elhajj, I. (2006) A Randomized Double-Blind Placebo-Controlled Trial of Rifaximin in Patients with Abdominal Bloating and Flatulence. The American Journal of Gastroenterology, 101, 326-333. https://doi.org/10.1111/j.1572-0241.2006.00458.x

[29] Ford, A., Moayyedi, P., Lacy, B., Lembo, A., Saito, Y., Schiller, L., et al. (2014) Task Force on the Management of Functional Bowel Disorders. American College of Gastroenterology Monograph on the Management of Irritable Bowel Syndrome and Chronic Idiopathic Constipation. The American Journal of Gastroenterology, 109, S2-S26. https://doi.org/10.1038/ajg.2014.187

[30] Menees, S., Maneerattannaporn, M., Kim, H. and Chey, W. (2012) The Efficacy and Safety of Rifaximin for the Irritable Bowel Syndrome: A Systematic Review and Meta-Analysis. The American Journal of Gastroenterology, 107, 28-35. https://doi.org/10.1038/ajg.2011.355

[31] Shah, E., Kim, S., Chong, K., Lembo, A. and Pimentel, M. (2012) Evaluation of Harm in the Pharmacotherapy of Irritable Bowel Syndrome. American Journal of Medicine, 125, 381-393.

[32] Pimentel, M., Chow, E.J. and Lin, H.C. (2003) Normalization of Lactulose Breath Testing Correlates with Symptom Improvement in Irritable Bowel Syndrome: A Double-Blind,Randomized, Placebo-Controlled Study The American Journal of Gastroenterology, 98, 412-419.

[33] Posserud, I., Stotzer, P., Björnsson, E., Abrahamsson, H. and Simrén, M. (2007) 
Small Intestinal Bacterial Overgrowth in Patients with Irritable Bowel Syndrome. Gut, 56, 802-808. https://doi.org/10.1136/gut.2006.108712

[34] Kassinen, A., Krogius-Kurikka, L., Makivuokko, H., et al. (2007) The Fecal Microbiota of Irritable Bowel Syndrome Patients Differs Significantly from That of Healthy Subjects. Gastroenterology, 133, 24-33.

https://doi.org/10.1053/j.gastro.2007.04.005

[35] Pimentel, M. (2009) Review of Rifaximin as Treatment for SIBO and IBS. Expert Opinion on Investigational Drugs, 18, 349-358. https://doi.org/10.1517/13543780902780175

[36] Brigidi, P., Swennen, E., Rizzello, F., Bozzolasco, M. and Matteuzzi, D. (2002) Effects of Rifaximin Administration on the Intestinal Microbiota in Patients with Ulcerative Colitis. Journal of Chemotherapy, 14, 290-295.

https://doi.org/10.1179/joc.2002.14.3.290

[37] Lotronex (Alosetron Hydrochloride) Tablets. Prometheus Laboratories, San Diego, 2008 (Package Insert).

[38] Zelnorm (Tegaserod Maleate) Tablets. Novartis Pharmaceuticals, East Hanover, 2007 (Package Insert).

[39] Amitiza (Lubiprostone) Capsules. Takeda Pharmaceuticals America, Deerfield, 2008 (Package Insert). 